\title{
Maintaining motor units into old age: running the final common pathway
}

\author{
Geoffrey A. Power (1), Brian H. Dalton (2), Kevin J. Gilmore (3), Matti D. Allen (4), \\ Timothy J. Doherty (5), Charles L. Rice $(3,6)$
}

\begin{abstract}
(1) Department of Human Health and Nutritional Sciences, College of Biological Sciences, University of Guelph, Guelph, Ontario, Canada; (2) School of Health and Exercise Sciences, The University of British Columbia Okanagan, Kelowna, British Columbia, Canada; (3) Canadian Centre for Activity and Aging, School of Kinesiology, Faculty of Health Sciences, The University of Western Ontario, London, Ontario, Canada; (4) Schools of Medicine and Kinesiology and Health Studies, Faculty of Health Sciences, Queen's University, Kingston, Ontario, Canada; (5) Departments of Physical Medicine and Rehabilitation and Clinical Neurological Sciences, Schulich School of Medicine and Dentistry, The University of Western Ontario, London, Ontario, Canada; (6) Department of Anatomy and Cell Biology, Schulich School of Medicine and Dentistry, The University of Western Ontario, London, Ontario, Canada.
\end{abstract}

This article is distributed under the terms of the Creative Commons Attribution Noncommercial License (CC BY-NC 4.0) which permits any noncommercial use, distribution, and reproduction in any medium, provided the original author(s) and source are credited.

\begin{abstract}
Invited Letter to the Editor. This article is a commentary on the recently published manuscript "Use it or lose it: tonic activity of slow motoneurons promotes their survival and preferentially increases slow fiber-type groupings in muscles of old lifelong recreational sportsmen". Mosole S, Carraro U, Kern H, Loefler S, Zampieri S. Use it or lose it: tonic activity of slow motoneurons promotes their survival and preferentially increases slow fiber-type groupings in muscles of old lifelong recreational sportsmen. Eur J Transl Myol 2016;26:5972. doi: 10.4081/ejtm.2016.5972. We offer some unique perspectives on masters athletes and the role of physical activity in maintaining the number and function of motor units into old age.
\end{abstract}

Key Words: Motor unit, masters athlete, EMG, motor neuron.

Eur J Transl Myol 2017;27(1):71-73

We read with great interest a recent article from Mosole et al. $^{1}$ that synthesized the literature and presented some new data to provide clarity on whether life-long exercise promotes reinnervation of muscle and survival of spinal [motor neurons (MNs)] and [motor units (MUs)] into old age. The authors reported very interesting and exciting data from both rodent and human models that investigated the age-related loss of MNs and the resulting effects on skeletal muscle. We would like to take this opportunity to provide support for the findings reported by Mosole et al. ${ }^{1}$ by integrating findings we have reported in masters athletes ${ }^{2-5}$ and to add to this valuable discussion.

A MU is composed of the MN with its cell body located in the ventral spinal cord, its motor axon, and the muscle fibres it innervates and is regarded as the final common pathway for motor control. Therefore, maintaining MUs into old age is important from both a functional and anatomical perspective. With electromyographic techniques it is possible in humans to estimate the number of functioning MUs [i.e., MU number estimate (MUNE)] and gain insight into the neurophysiological changes associated with age-related remodeling, such as the quality of MU neuromuscular transmission ${ }^{2}$. From these studies, a gradual reduction in the number of functioning MUs has been reported after the $3^{\text {rd }}$ decade of life until the $7^{\text {th }}$ decade followed by a rapid decline thereafter into very old age. ${ }^{6-8}$ Up to the $7^{\text {th }}$ decade, muscle mass appears to be maintained through the process of collateral reinnervation, whereby healthy MNs sprout axons and successfully reinnervate orphaned muscle fibers following the death of a parent MN. Life-long physical activity has been shown to protect against the typical age-related loss of MUs in most $^{2-4}$, but not all $^{9}$ studies in humans. However, in humans it is not entirely clear the 'optimal' type of exercise, or dose-response relationship needed to impart a neuroprotective effect. In old rodents, in studies that used synergist ablation, thus effectively overloading the muscle $^{10}$, there was significant compensatory muscle 
hypertrophy, but MN numbers within the spinal cord were similar to the control limb. Conversely, those rodents subjected to life-long swimming, ${ }^{11}$ maintained MN numbers well into old age. This neuroprotection of MNs in rodents was later corroborated in the tibialis anterior of masters athletes (average age of 65 and 80 years). ${ }^{2,4}$ Not necessarily surprising, there does not appear to be an overall systemic preservation of MUs in masters runners because MUNE in the biceps brachii (a less active muscle in runners) was similar to agematched controls. ${ }^{3}$ Together, most findings in humans appear to support results shown in animal models that chronic activation of the MN pool specific to the muscle exercised seems to be required for delaying the 'typical' age-related loss of MUs. The precise mechanisms by which chronic physical activity protects MUs from agerelated loss remains unclear. Future work using animal models should shed some light on appropriate therapies to maintain functional MUs during the aging process. In addition to the number of functional MUs, the integrity of neuromuscular transmission with adult aging can be assessed by measuring MU potential stability through measures of variability in the overall shape and timing of consecutively detected near fiber MU action potentials as measured using needle electromyography ${ }^{12,13}$. In world class octogenarian masters athletes we found a higher number of functioning MUs as compared with age-matched community dwelling controls. ${ }^{2}$ Of special interest was that the level of collateral reinnervation was not different (i.e., the negative peak amplitude of the [surface motor unit potential (SMUP)] representing the electrophysiological size of the remodeled motor unit was not different) between groups. Despite the same MU size, the masters athletes exhibited greater MU stability, as determined by their lower near fiber jiggle and jitter values (i.e. MU parameters used to assess MU transmission stability) ${ }^{12}$ compared with age-matched controls. The greater levels of MU potential stability in the masters athletes versus the controls may be reflective of protection against abnormalities in muscle fiber action potential propagation, or neuromuscular transmission instability due to the development of dysfunctional neuromuscular junctions of newly reinnervated fibres. ${ }^{14}$ Additionally, we reported increased near-fiber potential count in age-matched controls compared with masters athletes, which may be more sensitive than surface EMG measures (i.e., SMUP) in detecting reinnervation, presumably to compensate for prior denervation (i.e. motor unit remodeling). ${ }^{15}$ These electrophysiological measures of fiber grouping are consistent with histological evidence of increased MU homogeneity in advanced age. 1,14,16,17 Masters athletes have provided an interesting model in humans to explore healthy adult aging in a population free from many confounding factors known to accelerate the aging process such as obesity and immobility/disuse. Both masters athletes and healthy older adults seem to experience MU remodeling but the masters athletes exhibit less MU loss and more effective reinnervation and more stable neuromuscular transmission, whereas the age-matched controls presented with more extensive remodeling and less stable neuromuscular transmission. The presumed better maintenance of MUs in masters athletes occurs at a time point in the aging process when MU loss is greatest and the loss of muscle mass and strength becomes clinically relevant, potentially maintaining function and attenuating or delaying sarcopenia in this exceptional cohort of older adults. More studies are needed to relate these measures to functional tasks in humans. However, studies in animal models and humans such as reported by Mosole et al. ${ }^{1}$ are critical to provide further understanding and insight into the neurophysiology of aging and human neuromuscular function.

\section{Author's contributions}

All authors contributed to the writing and editing of the article.

\section{Acknowledgments}

The studies were supported by the Natural Sciences and Engineering Research Council of Canada (NSERC) and the Canadian Institutes of Health Research (CIHR).

\section{Conflict of Interest}

The authors declare no conflicts of interest.

\section{Corresponding Author}

Geoffrey A. Power, Ph.D.

Neuromechanical Performance Research Lab

Department of Human Health and Nutritional Sciences

College of Biological Sciences

University of Guelph, Ontario, Canada N1G 2W1

Phone: 519-824-4120 x53752

Email: gapower@uoguelph.ca

E-mails of coauthors

Brian H. Dalton: brian.dalton@ubc.ca

Kevin J. Gilmore: kgilmor2@uwo.ca

Matti D. Allen: mallen@qmed.ca

Timothy J. Doherty: tim.doherty@lhsc.on.ca

Charles L. Rice: crice@uwo.ca

\section{References}

1. Mosole S, Carraro U, Kern H, et al. Use it or Lose It: Tonic activity of slow motoneurons promotes their survival and preferentially increases slow fiber-type groupings in muscles of old Lifelong Recreational Sportsmen. Eur J Transl Myol 2016;26:5972. doi: 10.4081/ejtm.2016.5972

2. Power GA, Allen MD, Gilmore KJ, et al. Motor unit number and transmission stability in octogenarian world class athletes: Can age-related deficits be outrun? J Appl Physiol 2016;121:10131020 . 
3. Power GA, Dalton BH, Behm DG, et al. Motor unit survival in lifelong runners is muscle dependent. Med Sci Sports Exerc 2012;44:123542.

4. Power GA, Dalton BH, Behm DG, et al. Motor unit number estimates in masters runners: use it or lose it? Med Sci Sports Exerc 2010;42:1644-50.

5. Power GA, Dalton BH, Doherty TJ, et al. If you don't use it you'll likely lose it. Clin Physiol Funct Imaging 2016;36:497-498.

6. McComas AJ. Invited review: motor unit estimation: methods, results, and present status. Muscle Nerve 1991;14:585-97.

7. McNeil CJ, Doherty TJ, Stashuk DW, et al. Motor unit number estimates in the tibialis anterior muscle of young, old, and very old men. Muscle Nerve 2005;31:461-7.

8. Power GA, Allen MD, Booth WJ, et al. The influence on sarcopenia of muscle quality and quantity derived from magnetic resonance imaging and neuromuscular properties. Age 2014;36:13771388.

9. Piasecki M, Ireland A, Coulson J, et al. Motor unit number estimates and neuromuscular transmission in the tibialis anterior of master athletes: evidence that athletic older people are not spared from agerelated motor unit remodeling. Physiol Rep 2016;4.

10. Kanda K, Hashizume K, Miwa T, et al. Overloading a muscle does not alter the rate of motoneuronal loss in aged rats. Neurobiol Aging 1996;17:613-7.

11. Kanda K and Hashizume K. Effects of long-term physical exercise on age-related changes of spinal motoneurons and peripheral nerves in rats. Neurosci Res 1998;31:69-75.

12. Stalberg EV, Sonoo M. Assessment of variability in the shape of the motor unit action potential, the "jiggle," at consecutive discharges. Muscle Nerve 1994;17:1135-44.

13. Stashuk DW. Detecting single fibre contributions to motor unit action potentials. Muscle Nerve 1999;2:218-29

14. Hepple RT, Rice CL. Innervation and neuromuscular control in ageing skeletal muscle. J Physiol 2016;594:1965-78.

15. Bril V, Werb MR, Greene DA, et al. Single-fiber electromyography in diabetic peripheral polyneuropathy. Muscle Nerve 1996;19:2-9.

16. Lexell J, Downham D, and Sjostrom $M$. Distribution of different fibre types in human skeletal muscles. Fibre type arrangement in $\mathrm{m}$. vastus lateralis from three groups of healthy men between 15 and 83 years. J Neurol Sci 1986;72:211-22.

17. Kanda $\mathrm{K}$ and Hashizume K. Changes in properties of the medial gastrocnemius motor units in aging rats. J Neurophysiol 1989;61:737-46. 